Nervenarzt 2009 [Suppl 1] $\cdot 80: 26-26$ DOI 10.1007/s00115-009-2781-6

(c) Springer Medizin Verlag 2009
T. Ziemssen $\cdot$ K. Thomas $\cdot$ M. Marggraf $\cdot$ T. Schultheiss

Neuroimmunologisches Labor, MS Zentrum, Neurologische Universitätsklinik, Dresden

\title{
Regulatorische T-Zellen bei Patienten mit Multipler Sklerose
}

\section{Einleitung}

Regulatorische T-Zellen scheinen in der Pathogenese autoimmuner Erkrankungen eine wichtige Rolle zu spielen. So konnte bei Patienten mit schubförmig-remittierender Multipler Sklerose (RRMS), nicht aber mit sekundär progredienter MS, gezeigt werden, dass $\mathrm{CD}_{4}{ }^{+}$-CD25(high)-regulatorische T-Zellen (Tregs) eine reduzierte suppressive Funktion aufweisen.

\section{Patienten und Methoden}

In dieser Studie wurde die Expression des Transkriptionsfaktors $\mathrm{FOXP}_{3}$ mittels intrazellulärer Durchflusszytometrie, der so genannten FACS-Analyse, untersucht, um regulatorische T-Zellen zu identifizieren. Es wurden 20 MS-Patienten mit schubförmig-remittierender Verlaufsform vor und nach immunmodulatorischer The- rapie mit Interferon (IFN) $\beta-1 b$ sowie 10 gesunde Kontrollen untersucht. Zusätzlich wurden die Häufigkeiten von unterschiedlichen dendritischen Zellpopulationen und Makrophagen in den beiden Gruppen bestimmt.

\section{Ergebnisse}

Im Vergleich zu den Kontrollen wiesen MS-Patienten eine geringere Anzahl von $\mathrm{CD}_{4}{ }^{+}-\mathrm{CD}_{25}$ (high)-FOXP $3{ }^{+}-\mathrm{T}-$ Zellen auf, die mit einer reduzierten Suppressionskapazität der Tregs verbunden war. Eine Therapie mit IFN $\beta$-1b führte zu einer Zunahme der Anzahl der FOXP $3{ }^{+}-\mathrm{T}-$ Zellen (• Abb. 1), sodass T-Effektor-Zellen (Teff) stärker inhibiert werden können (• Abb. 2). Es konnten keine Unterschiede hinsichtlich der Häufigkeiten dendritischer Zellpopulationen bzw. Makrophagen nachgewiesen werden.

\section{Schlussfolgerungen}

Zusammenfassend konnte eine aberrante $\mathrm{FOXP}_{3}$-Expression bei Patienten mit RRMS nachgewiesen werden. Eine immunmodulatorische Therapie mit IFN $\beta$ $1 \mathrm{~b}$ führte zu einer Zunahme der Tregs und stellte zudem das Ausmaß der Teff-Inhibierung auf nahezu Kontrollniveau wieder her.

\section{Korrespondenzadresse}

PD Dr. med. T. Ziemssen

Neuroimmunologisches Labor, MS Zentrum, Neurologische Universitätsklinik Fetscherstraße 74, 01307 Dresden Tjalf.Ziemssen@uniklinikum-dresden.de

Interessenkonflikt. Der korrespondierende Autor gibt an, dass über die Interessenkonflikte „Vertragsho-

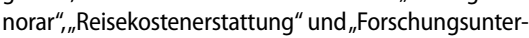
stützung durch die Bayer Vital GmbH/Bayer Schering Pharma AG kein weiterer Interessenkonflikt besteht.

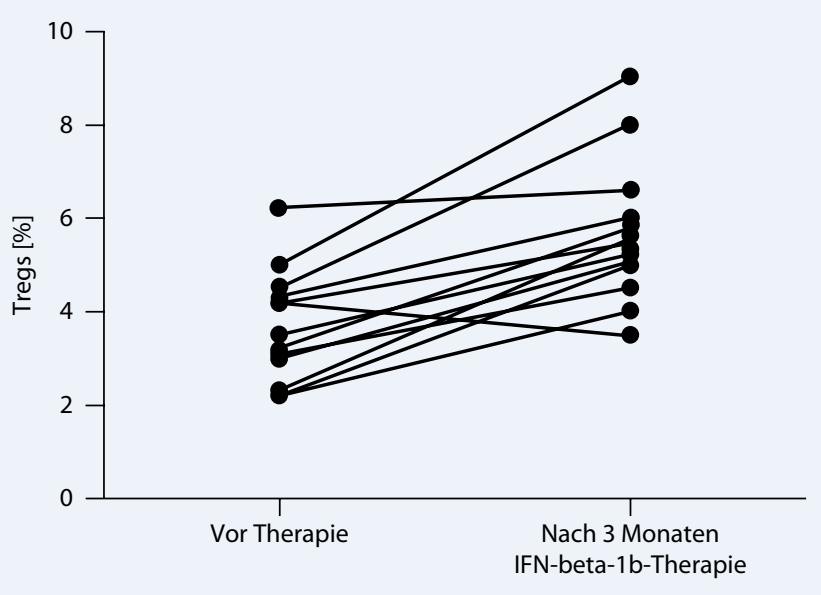

Abb. $1 \Delta$ IFN $\beta$-1b steigert die Frequenz regulatorischer T-Zellen (Tregs; $\mathrm{CD}^{+} \mathrm{CD} 25$ (high) FOXP3 ${ }^{+}$) nach 3 Monaten Therapie bei MS-Patienten $(n=15)$

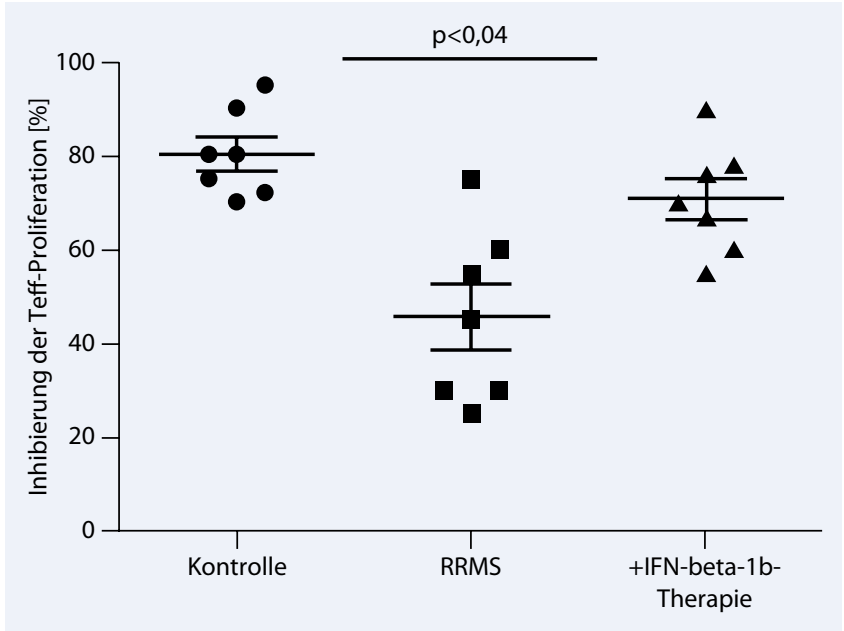

Abb. $2 \Delta$ IFN $\beta$-1b stellt das bei RRMS-Patienten signifikant erniedrigte Ausmaß der T-Effektor-Zell-(Teff)-Inhibition von regulatorischen T-Zellen (Tregs) auf nahezu Kontrollniveau wieder her 